\title{
SIMULINVE - UM SIMULADOR DE INVENTÁRIO PARA UM CENTRO DE DISTRIBUIÇÃO DE PEÇAS
}

\section{SIMULINVE - A SIMULATOR OF THE INVENTORY OF A PART DISTRIBUTION CENTER}

\author{
Heráclito Lopes Jaguaribe Pontes \\ Doutorando em Engenharia Mecânica \\ Universidade de São Paulo - Escola de Engenharia de São Carlos \\ Departamento de Engenharia Mecânica \\ Telefone: (16) 3373-9432 E-mail: hjpontes@sc.usp.br \\ Jandira Guenka Palma \\ Professor Adjunto \\ Universidade Estadual de Londrina - Centro de Ciências Exatas \\ Departamento de Computação
}

Avenida Trabalhador Sãocarlense, 400 - Centro, São Carlos-SP - Brasil - 13566-590

Rod. Celso Garcia, s/n, km 380 - Campus Universitário, Londrina-PR - Brasil - 86051-990

Telefone: (43) 3371-4678 E-mail: jgpalma@uel.br

\section{Arthur José Vieira Porto \\ Professor Titular}

Universidade de São Paulo - Escola de Engenharia de São Carlos

Departamento de Engenharia Mecânica

Avenida Trabalhador Sãocarlense, 400 - Centro, São Carlos-SP - Brasil - 13566-590

Telefone: (16) 3373-9432 E-mail: ajvporto@sc.usp.br

\section{RESUMO}

No mercado globalizado atual, as empresas buscam garantir disponibilidade de produto ao cliente final, com o menor nível de inventário possível. As ferramentas de simulação disponibilizam aos gestores melhores visões do negócio e melhores condições para a tomada de decisão. Em um Centro de Distribuição de Peças (CDP), para se alcançar um melhor desempenho no gerenciamento do inventário é importante poder contar com uma ferramenta capacitada para realizações de simulações de possíveis cenários. Este trabalho teve como 
objetivo desenvolver um processo para gerenciamento de inventario que resultou em um simulador de inventário para CDP. O simulador realiza projeções visando obter como resultados a previsão de demanda, a política de reposição das peças, o percentual de atendimento dos pedidos, os meses de inventário, a quantidade de Scrap e a quantidade de peças. $\mathrm{O}$ simulador foi submetido a experimentos com três cenários diferentes e os resultados confirmaram a qualidade do sistema de simulação proposto.

Palavras-chave: Centro de Distribuição de Peças, Inventário, Simulação.

\begin{abstract}
In the current global market, the companies are trying to become their product available to the final client with the smallest possible level of inventory. The simulation tools offer to managers best views of business and best conditions to decide. In a Part Distribution Center (PDC), to reach the best performance in the management of the inventory, it is important to be able to count on a tool that is capable of accomplishments from simulation possible sceneries. This work had as objective to develop a process for inventory management which resulted in a simulator of the inventory for a PDC. The simulator performs projections in order to get demand forecasts, the parts replenishment policy, the attendance percentage, the inventory months, scrap quantity and quantity part to devolution. The simulator was submitted to experiments with three different sceneries and the results confirmed the quality of the simulation system proposed.
\end{abstract}

Key-words: Part Distribution Center, Inventory, Simulation.

\title{
1. INTRODUÇÃO
}

No mercado globalizado, onde os clientes exigem cada vez mais qualidade, confiabilidade e preço de produtos e serviços, as empresas têm se preocupado em desenvolver formas para ganhar vantagens sobre os seus concorrentes. As empresas procuram agilizar o fluxo de materiais, comprimindo o tempo entre o recebimento e a entrega dos pedidos, para reduzir os investimentos em inventário.

Neste ambiente competitivo entre as empresas, o papel da armazenagem está voltado para prover capacidade de resposta rápida e muitos dos serviços executados visam reduzir as necessidades de grandes quantidades de peças no inventário. Acompanhando esse cenário, o mercado está migrando para a centralização de estoque, fazendo com que os Centros de Distribuição (CD) ou mais especificamente os Centros de Distribuição de Peças (CDP) assumam um papel de relevância logística (VAN DE BERG E ZIJM, 1999). 
Os CDP são instrumentos que viabilizam de forma competitiva o fluxo de mercadorias vindas dos fabricantes, até os seus diversos graus de capilaridade distributiva. A volatilidade econômica, característica do mercado contemporâneo, resulta em uma maior complexidade gerencial para os CDP devido a: pedidos mais frequientes e em quantidades menores, mudanças no mix de produtos e a competição baseada no ciclo do pedido e na qualidade. Os CDP, diante dos fatores citados, precisam de sistemas de controle gerencial de inventário com alto grau de precisão e de ferramentas para prever o comportamento do inventário no futuro (FARAH JUNIOR, 2002).

A eficácia do gerenciamento do inventário tem grande influência na lucratividade do CDP e na empresa como um todo. A capacidade de gerenciar o inventário determina diretamente os níveis de estoques necessários para atingir os níveis de serviço desejados. Em muitas empresas, os estoques representam o maior valor do ativo, ou seja, melhorar o desempenho dos estoques resulta em importantes melhorias no fluxo de caixa e em aumentos da lucratividade.

O simulador proposto tem a finalidade de trabalhar com suposições, projeções e manipulações de variáveis, assim, permite ao operador do inventário investigar os resultados conforme as variações dos prováveis acontecimentos. O simulador possibilita aos responsáveis pelo inventário avaliar diferentes políticas gerenciais sem impactar ou interromper os processos em andamento no dia-a-dia do CDP.

O presente trabalho é classificado como uma pesquisa do tipo estudo de caso, devido ao fato de envolver o estudo do inventário em um Centro Distribuição de Peças específico.

A empresa utilizada como base para o desenvolvimento do simulador de inventário é uma multinacional localizada no estado de São Paulo. Em seu CDP no Brasil ocorrem atualmente cerca de 50.000 Calls (atendimentos) por mês e para atender esta demanda o inventário do CDP é composto por aproximadamente 100.000 peças.

\section{REVISÃO BIBLIOGRÁFICA}

Para o desenvolvimento do simulador de inventário para o CDP proposto neste trabalho, foi necessário o entendimento de vários conceitos que são:

- Centro de Distribuição de Peças: sua definição, funções e benefícios; 


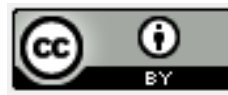

- Inventário: sua definição, como gerenciar o inventário e principais medidas de desempenho;

- Simulação: sua definição, suas vantagens e desvantagens e quando utilizá-la.

\section{Centro de Distribuição de Peças (CDP)}

O Centro de Distribuição de Peças (CDP) é o local onde se armazena peças de um fabricante ou de diversos fabricantes por um determinado período de tempo, para serem consolidados e enviados aos seus consumidores em comum (FRAZELLE, 2002).

Para Mulcahy (1994), CDP é definido como um espaço físico responsável pela armazenagem de uma variedade de peças de um fornecedor ou da própria fábrica e responsável também pela liberação das peças para seus clientes. Conforme Rodrigues e Pizzolato (2003), o conceito de CDP é moderno e suas funções ultrapassam as tradicionais funções dos depósitos, galpões ou almoxarifados, as quais não são adequadas dentro do sistema logístico.

Segundo Farah Junior (2002), os CDP são instrumentos que viabilizam de forma competitiva o fluxo de peças vindas dos fabricantes, até os seus diversos graus de capilaridade distributiva. O CDP deve ser um meio de minimização de custos, melhoria no uso dos recursos e apoio ao processo de venda e pós-venda.

De acordo com Tompkins et al. (1996), existem diversos tipos de armazéns dentre eles o CDP. De acordo com a localização destes armazéns no contexto logístico, estes podem ter diferentes funções. Em geral, as funções dos CDP são:

- Permitir o equilíbrio entre a quantidade produzida e a demanda;

- Diminuir a distância de transporte para permitir uma resposta aos clientes em menor tempo;

- Permitir a consolidação de produtos de vários fornecedores para consumidores comuns, ou seja, tornar os produtos mais próximos à manufatura e ao consumo.

Diversas vantagens são identificadas na literatura quanto à adoção do CDP no sistema logístico. Essas vantagens obtidas pela armazenagem, centralização de estoque podem beneficiar todos os elos da cadeia: fornecedor, empresa e consumidor.

Segundo Farah Junior (2002), algumas vantagens de um CDP são: cumprimento do tempo de entrega, precisão no atendimento, qualidade do produto entregue, suporte no pósvenda, redução do custo de transporte, liberação de espaço nas fábricas e nos clientes, redução 
de mão-de-obra nos clientes para o recebimento e conferência de mercadorias e diminuição de falta de produtos nos clientes.

Bowersox e Closs (2001) identificam também duas vantagens na adoção do CDP no sistema logístico: a capacidade de agregar valor ao produto (postergação) e os diferentes tipos de operações que podem ser realizadas no mesmo (Consolidação de cargas, Break Bulk, Cross-Dock e Formação de Estoque).

\subsection{Inventário}

Segundo Cox III e Blackstone (2002), inventário são aqueles estoques usados para suportar produção (matéria-prima e itens em processo), suportar atividades gerais (manutenção, reparos e suprimento de operações) e suportar serviço aos clientes (produto acabado, partes e peças).

$\mathrm{O}$ inventário consiste em uma reserva de matérias-primas, materiais em processo e produtos acabados ou peças que se encontram em inúmeros lugares dentro de uma empresa e em canais logísticos.

De acordo com Dear (1990), a classificação do inventário em grupos para o gerenciamento tem como objetivo tratar cada grupo de maneira diferente. As principais classificações são feitas em relação: aos fornecedores; a importância do item e classes ABC.

Conforme Krajewski e Ritzman (2004), o gerenciamento de inventário é uma preocupação importante para os gerentes em todos os tipos de empresa. Por essa razão, os gerentes acompanham de perto os inventários para mantê-los em níveis aceitáveis. O desafio consiste não em diminuir os estoques de forma excessiva para reduzir custos ou ainda ter muito estoque disponível a fim de satisfazer todas as demandas, mas em possuir a quantidade certa para alcançar as prioridades competitivas da empresa do modo mais eficiente.

A função do gerenciamento de inventário consiste em compatibilizar os custos e as pressões conflitantes que exigem estoques reduzidos e níveis de serviços elevados (KRAJEWSKI e RITZMAN, 2004).

Segundo Brewer et al. (2001), as organizações têm que responder três questões importantes no gerenciamento de inventário que são: que itens devem ser estocados, quando deve ser realizado um novo pedido do item e qual tamanho deve ser o pedido.

Segundo Forgaty et al. (1991), os dois principais critérios de avaliação do desempenho do gerenciamento de inventário são: 
- O nível de serviço prestado ao cliente;

- O investimento necessário em inventário para alcançar esse nível de serviço.

De acordo com Bowersox e Closs (2001), o nível de serviço comporta objetivos de desempenho que a função de estoque deve ser capaz de cumprir, ou seja, o nível de serviço define quais os objetivos de desempenho que os estoques devem ter no atendimento das necessidades do mercado.

Conforme Gaither e Frazier (2002), nível de serviço refere-se à probabilidade de que um stockout (falta de estoque) não ocorrerá durante o lead time. O nível de serviço é um objetivo fixado pela alta administração. Comporta objetivos de desempenho que a função de estoque deve ser capaz de cumprir.

Ao gerenciar o inventário é importante manter as atenções voltadas para a questão da quantidade de peças no inventário, haja vista que, quando diminui a quantidade física de peças no CDP, gera-se uma economia de área, menos movimentação, menor custo de armazenagem, menor custo de administração menor custo de obsolescência, dentre outros.

De acordo com Slack et al. (1997), meses de inventário ou cobertura do número de meses do estoque é a quantidade de tempo que o estoque duraria, sujeito à demanda real, se não fosse reabastecido.

Conforme Krajewski e Ritzman (2004), número de meses de suprimento é uma medida de estoque obtida dividindo-se o valor em meses do estoque agregado médio pelas vendas mensais a preço de custo.

\subsection{Simulação}

Simulação computacional ou simulação é o processo de projetar um modelo lógico matemático de um sistema real e fazer experimentos deste sistema no computador (PRITSKER, 1986).

Conforme Shannon (1998), simulação consiste no processo de desenvolver um modelo de um sistema real e realizar experimentos com este modelo com o propósito de entender o comportamento do sistema e/ou as evoluções das várias estratégias para a operação do sistema.

Para Harrel e Tumay (1997), simulação é uma atividade por meio da qual se pode tirar conclusões sobre o comportamento de um dado sistema pelo estudo do comportamento do seu 


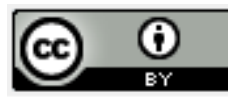

modelo correspondente no qual as relações de causas e efeitos são as mesmas ou simulares do sistema real.

Segundo Freitas Filho (2001), a simulação permite ao analista realizar estudos sobre os correspondentes sistemas para responder questões do tipo "O que aconteceria se". O principal apelo ao uso desta ferramenta, é que tais questões podem ser respondidas sem que os sistemas sob investigação sofram qualquer perturbação, uma vez que os estudos são realizados no computador. A simulação permite que tais estudos sejam realizados sobre sistemas que ainda não existem, levando ao desenvolvimento de projetos eficientes antes que qualquer mudança física tenha sido iniciada.

Bertrand e Fransoo (2002) apontam que apesar da qualidade científica dos resultados da simulação ser menor que o caso de análise matemática, sua relevância cientifica é alta. A variedade de modelos científicos na qual a simulação é capaz de trabalhar é maior que uma análise matemática. Eles apontam que a simulação é utilizada em casos onde os modelos ou problemas são muitos complexos para uma análise matemática formal.

As vantagens da simulação são citadas por Banks (1998), Banks, Carlson e Nelson (1996), Kelton et al. (1998), Carson II (2004) e Centeno e Carrillo (2001) são:

- Modelos mais realistas: maior liberdade na construção do modelo;

- Processo de modelagem evolutivo;

- Uma vez criado, um modelo pode ser utilizado inúmeras vezes para avaliar projetos e políticas propostas;

- Os modelos de simulação podem ser quase tão detalhados quanto os sistemas reais, novas políticas e procedimentos operacionais, regras de decisão, fluxos de informação, podem ser avaliados sem que o sistema real seja perturbado;

- Perguntas do tipo "e se?" (“what if?”): em diversos casos, em lugar de buscar uma solução, o objetivo resume-se em tornar mais claras as possíveis consequiências de um conjunto de decisões;

- Aplicação a problemas mal-estruturados;

- Aquisição de visão sistêmica.

Os mesmos autores citam também algumas desvantagens da simulação:

- A modelagem e a análise da simulação podem ser dispendiosas em termos de recursos financeiros e de tempo;

- A construção de modelos requer treinamento especial; 
- A programação de um modelo de simulação pode ser tornar altamente dispendiosa e desgastante se os recursos computacionais não forem apropriados;

- Os resultados da simulação são, muitas vezes, de difícil interpretação;

\section{SIMULINVE - UM SIMULADOR DE INVENTÁRIO PARA UM CDP}

\subsection{Definição do Simulinve}

O simulador desenvolvido neste trabalho complementa o sistema gerencial de inventário de um CDP realizando simulações futuras dos indicadores gerenciais sem agredir a integridade dos dados contidos no sistema gerencial já existente. A partir destas simulações, os responsáveis pelo inventário têm condições de tomar decisões com maior segurança e mais rapidamente. $\mathrm{O}$ simulador possibilita aos mesmos avaliar diferentes políticas gerenciais sem impactar ou interromper os processos em andamento no dia-a-dia do CDP.

\subsection{Metodologia de Desenvolvimento do Simulinve}

O desenvolvimento do simulador foi baseado numa adaptação da metodologia de simulação desenvolvida por Freitas Filho (2001) que foi dividida nos seguintes passos: formulação e análise do problema; planejamento do projeto; formulação do modelo conceitual; coleta de informações e dados; tradução do modelo; verificação e validação; experimentação; análise e interpretação dos resultados; e documentação.

A formulação e análise do problema iniciaram-se definindo os propósitos e objetivos do simulador de modo a entender quais parâmetros influenciam o sistema e o que está se modelando. Para isso traduziu-se a linguagem do operador do inventário para uma linguagem precisa e claramente entendida. Inicialmente, os esforços foram direcionados para o entendimento do sistema real. Para obtenção da familiarização e conhecimento adequado dos processos do inventário do CDP, todo o processo gerencial foi descrito em forma matemática/ lógica e em forma de fluxogramas. Após o período de familiarização com o sistema real confirmou-se a necessidade do desenvolvimento de uma ferramenta de apoio ao sistema de gerenciamento de inventário. Observou-se a necessidade de fazer projeções, manipular variáveis para ver como o inventário se comportaria em determinada situação. Neste momento foi respondida a pergunta sobre o porquê da existência deste simulador. No início do trabalho 
foi definido que não era esperado como resposta desta ferramenta decisões de otimizações, mas sim fazer simulações do sistema real. A ferramenta se limita a realizar projeções futuras a partir de dados fornecidos pelo sistema gerencial do inventário e de dados fornecidos na interface da ferramenta pelo operador do inventário.

No planejamento do projeto foram verificados os recursos (hardware e software) para realização do simulador proposto. Para melhor organização do desenvolvimento do projeto, foi construído um cronograma temporal das atividades envolvidas no sistema.

A formulação do modelo conceitual foi realizada sob o paradigma de orientação a objeto, empregando a UML (Unified Modeling Language) através do software Rational Rose®. Foi utilizado a UML para modelagem devido ao seu potencial na representação visual do sistema orientado a objeto.

$\mathrm{Na}$ coleta de informações e dados foi utilizada a técnica da análise documental. Os dados e informações relacionados à lógica de funcionamento do programa de gerenciamento do inventário do CDP em estudo foram coletados em forma de documentos escritos. Algumas informações e dados secundários foram solicitados durante o desenvolvimento do simulador também em forma de documentos escritos.

A tradução do modelo foi realizada na linguagem de programação $\mathrm{C}++($ Borland $C++$ Builder $\left.{ }^{\circledR}\right)$. Utilizou-se a linguagem de programação $\mathrm{C}++$ devido atender o nível de detalhamento necessário para representar o gerenciamento do inventário, considerando os limites de tempo de desenvolvimento, recursos financeiros e esforços de programação.

$\mathrm{Na}$ verificação fez-se uso das técnicas para identificação de problemas em programas computacionais, que são: a verificação por partes e o teste por parâmetros. A verificação por partes foi amplamente utilizada como forma de rastreamento e refinamento progressivo do modelo. Ela ocorreu durante a construção do simulador e, a cada parte construída, o simulador foi testado contra erros com o intuito de evitar a etapa posterior de correção do mesmo como um todo. O teste por parâmetros consistiu na execução da simulação através da variação dos parâmetros de entrada, verificando se os resultados obtidos apresentam um comportamento razoável.

A validação foi feita de duas formas: pela comparação das saídas do simulador e do sistema real e pela análise de especialistas. O simulador foi validado por especialistas com profundos conhecimentos acerca do sistema gerencial de inventário do CDP. 
Para experimentação do simulador foram desenvolvidos três cenários com diferentes dados de entrada e horizontes de simulação (seis, dez e doze meses). A quantidade de peças simuladas foi à mesma para os três cenários (1.000 peças).

Os resultados obtidos pelos três cenários simulados foram condizentes com o sistema real. O horizonte de simulação não influenciou nos resultados finais, ou seja, para o simulador não importa a quantidade de meses que se está simulando, mas sim os parâmetros de entrada. Com os resultados deste trabalho comprovou-se que o simulador condiz com a realidade, assim o mesmo esta pronto para fazer projeções futuras e o resultado estará condizente desde que os dados de entradas correspondam ao histórico, e avaliações de mercado atuais e projetadas correspondam realmente aos acontecimentos futuros.

A documentação foi realizada de duas formas: no próprio simulador e em um relatório de progresso. Durante o desenvolvimento foi documentada cada versão do simulador de acordo com o seu desenvolvimento e no próprio simulador comentou-se todas as atividades, para permitir o rápido e claro entendimento por outros usuários não familiarizados com o programa. Simultaneamente ao desenvolvimento foi criado um relatório com a descrição das atividades, fluxos, diagramas e a lógica. Neste relatório também estava à cronologia do trabalho e as principais decisões tomadas.

\subsection{Dados de Entrada do Simulinve}

O simulador possui duas formas de entrada de dados: por interface e por planilha do Microsoft Excel®. As entradas por interface são divididas em dois grupos: interface geral (variáveis de entrada utilizadas por todas as peças) e interface fonte (variáveis de entrada que diferem de acordo com o local de origem da peça, ou seja, nacional ou importada).

A entrada por arquivo (planilha do Microsoft Excel®) é realizada devido à necessidade de utilização no simulador de variáveis de entrada com valores individuais para cada peça, ou seja, cada peça tem características individuais que entram no programa pelo arquivo peça.

Os dados de entrada por interface são determinados pelo operador do inventário e os dados de entrada por planilha do Microsoft Excel® são fornecidos diretamente do sistema de gerenciamento de inventário do CDP.

Os principais dados de entrada por interface geral são: mês atual; horizonte de simulação; dias úteis dos meses de simulação; taxa do dólar; previsão da variação do 


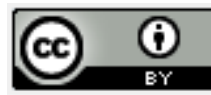

mercado; previsão de promoção de vendas; prazo máximo de permanência no CDP; freqüência para devolução das peças e valor disponível para devolução de peças.

Os principais dados de entrada por interfaces fonte são: estoque em trânsito; tempo de consolidação de pedidos; política de estoque máximo; política de estoque mínimo; lead time; e coeficiente de suavização. O coeficiente de suavização determina o equilíbrio entre a sensibilidade das previsões às mudanças na demanda e a estabilidade das previsões (TUBINO, 2000).

Os principais dados de entrada por planilha do Microsoft Excel® são: código da peça; código do inventário; lote mínimo de compra; múltiplo de embalagem; peso unitário; custo unitário; preço de venda; backorder (ordens em atraso); família da peça; estoque físico das peças; previsão de demanda para o primeiro mês de simulação; desvio para o primeiro mês de simulação; a demanda histórica de calls (atendimentos) dos últimos 36 meses; e quantidade de peças por calls dos últimos 36 meses.

\subsection{A lógica de Funcionamento do Simulinve}

O Simulinve executa seqüencialmente e de maneira repetitiva um conjunto de instruções. Na medida em que as instruções são executadas, os valores das variáveis são alterados, uma vez que se modificam as condições que influenciam o comportamento do simulador.

O simulador inicia quando o operador do inventário solicita acesso ao sistema. O sistema verifica se ele tem autorização, em caso positivo é liberado o acesso ao sistema. Se a autorização for negativa, o operador solicita login e senha para o administrador do sistema. Após entrar no simulador, o operador vai ao menu inventário e cria os arquivos de entrada de dados: fonte nacional, fonte importada e geral. Ainda no mesmo menu, o operador seleciona a opção simular e após abrir todos os arquivos de entrada (fonte nacional e importada, geral e arquivo peça), inicia-se a simulação.

A simulação inicia com a criação de uma lista de peças para inserção dos dados de cada peça. Em seguida, o código de cada peça é formado. De acordo com o código formado a peça é classificada. Os dados de entrada de cada peça são inseridos na lista de peças de acordo com a classificação da peça. Após a sua classificação, o sistema inicia o cálculo da projeção da demanda real da peça. 


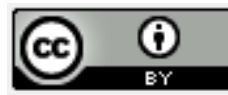

Depois de classificar as peças, projetar suas demandas reais para todo o horizonte de simulação, inicia-se a próxima fase da simulação em que o sistema realiza o cálculo da Reposição de Peças, o cálculo do Percentual de Atendimento de Pedidos, o cálculo dos Meses de Inventário, o cálculo do Scrap, o cálculo da Devolução de Peças e o cálculo da Previsão de Demanda para cada peça do inventário. O horizonte de simulação determina a quantidade de vezes que vão ser executadas estas operações. Após a simulação gera-se um arquivo de resultados com os dados de cada peça na extensão do horizonte de simulação.

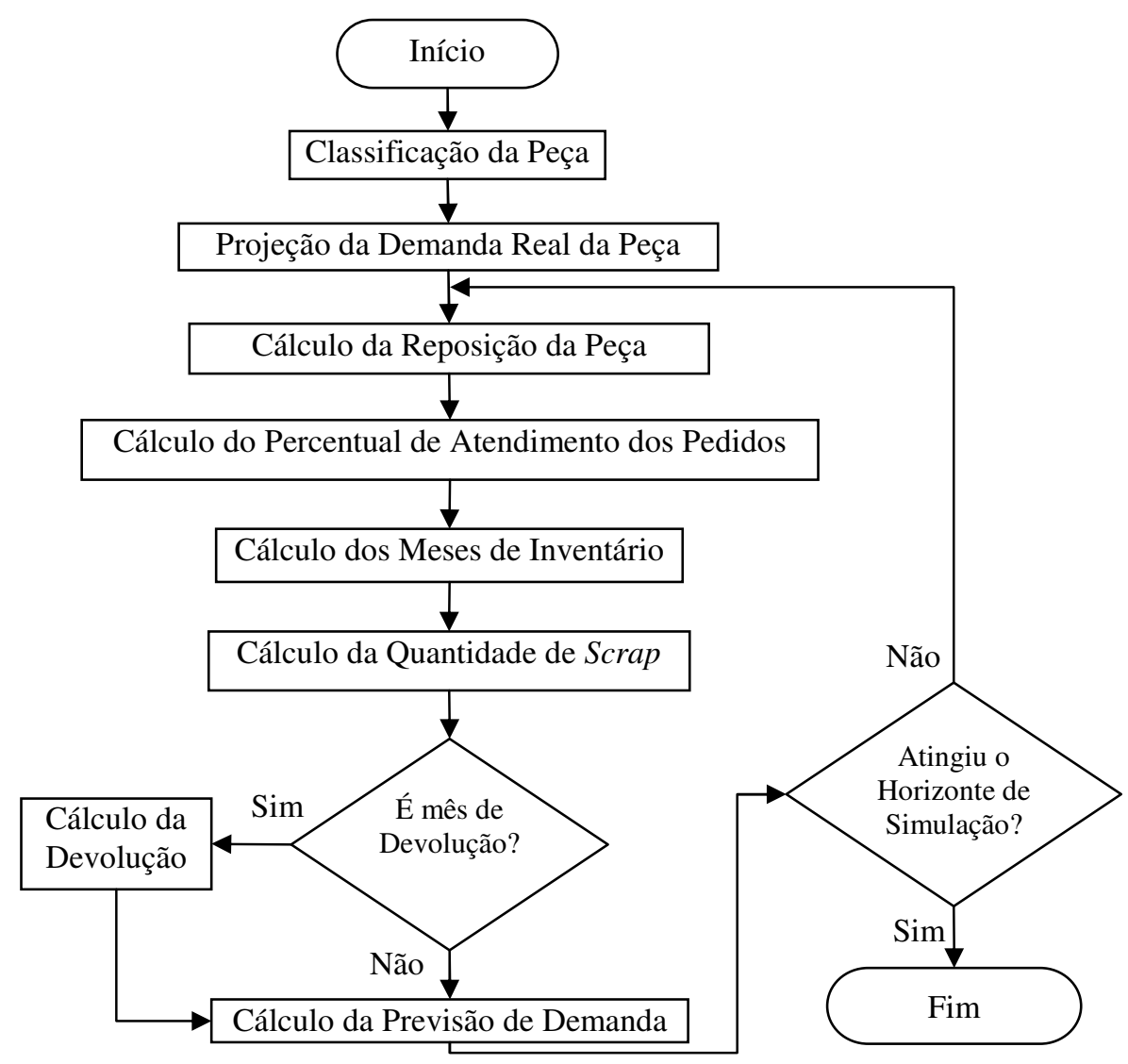

Figura 1 - Fluxograma Lógica de Funcionamento do Simulinve

\subsubsection{Classificação das Peças}

O código da peça é formado a partir da entrada dos dados pela planilha do Microsoft Excelß. Após a formação do código inicia-se a classificação. A classificação da peça consiste nas seguintes etapas:

- Primeiramente, verifica-se se a peça é desimpactada ou impactada. A peça é classificada como desimpactada quando o número de Calls (atendimentos) nos últimos 
doze meses é menor que três, ou seja, a peça não tem mais uma demanda significativa, com isso será descontinuada;

- O segundo passo da classificação é verificar qual a fonte da peça, nacional ou importada. As peças nacionais são divididas em dois tipos: peças produzidas na própria fábrica da empresa e as peças compradas de fornecedores nacionais;

- O último passo da classificação da peça é a verificação de qual classe a peça pertence. As classes são divididas em A, B, C. A classe de cada peça está relacionada com a quantidade de Calls dos últimos doze meses. Se nos últimos doze meses a peça tiver menos do que seis Calls, a peça é classificada como C. Se nos últimos doze meses a peça tiver mais de cinco e menos de trinta e seis Calls, a peça é classificada como B. Se nos últimos doze meses a peça tiver mais do que trinta e cinco Calls, a peça é classificada como A. Uma outra classificação realizada é a Normal e Proteção (proteção são peças que ficam estocadas no inventário devido ao lançamento de novas máquinas no mercado).

\subsubsection{Projeção da Demanda Real}

Para cada mês do horizonte de simulação é necessário ter uma demanda, como não é possível obter a demanda real de meses futuros, optou-se por calcular a projeção da demanda real baseando-se em métodos quantitativos baseados em séries temporais.

O cálculo da demanda real consiste em a partir da demanda histórica de trinta e seis meses projetar a demanda de acordo com horizonte de simulação. Essa projeção é realizada considerando diversos fatores como tendência, sazonalidade, promoção de venda, crescimento ou diminuição de mercado.

O cálculo da projeção da demanda real para cada peça é realizado da seguinte forma:

- Inicialmente calcula-se a tendência histórica (TH) baseada em trinta e seis meses de demanda histórica (DH). A tendência histórica é composta pela média móvel mensal (MM), o acréscimo ou decréscimo mensal da demanda (ADM) e o número de meses do horizonte de simulação $(\mathrm{k})$. O cálculo da demanda histórica $(\mathrm{DH})$ é obtido pela equação 3.1 .

$D H_{i}=C_{i} \times Q P C_{i}, i=1,36$ 


\section{Revista Produç@o}

A partir dos valores obtidos pela Eq.(3.1), soma-se as demandas históricas da seguinte forma:

$$
S D H_{i}=\sum_{j=i}^{i+11} D H_{j}, i=1,25
$$

A partir dos valores obtidos na Eq.(3.2), tem-se:

$$
M M_{i}=\frac{\left(S D H_{i}+S D H_{i+1}\right)}{24}, i=1,24
$$

A partir dos valores obtidos na Eq. (3.3), compara-se se houve acréscimo ou decréscimo na demanda histórica:

$$
A D M=\sum_{i=13}^{24} M M_{i}-\sum_{i=1}^{12} M M_{i}
$$

Somando a Eq.(3.3) com a Multiplicação da Eq. (3.4) pelo número de meses do horizonte de simulação (k), tem-se:

$$
T H=M M_{24}+(k \times A D M)
$$

Onde:

DH - demanda histórica

C - número de Calls

QPC - número de peças por Call

SDH - soma da demanda histórica utilizando doze meses seqüenciais

$\mathrm{TH}$ - tendência histórica

k - número de meses do horizonte de simulação

- A segunda etapa é o cálculo da sazonalidade (S). A sazonalidade é obtida pelo cálculo do desvio médio real (DMR). O DMR é obtido a partir do cálculo do desvio real da média móvel (DR). As equações 3.6 e 3.7 mostram o cálculo de DR, DMR e S;

$$
\begin{aligned}
& D R=\left(\frac{D H_{i}}{M M_{i}}\right) \times 100, i=1,24 \\
& S=D M R_{j}=\left(\frac{D R_{j}+D R_{j+12}}{2}\right), j=1,12
\end{aligned}
$$


Onde:

DR - desvio real da média móvel

$\mathrm{S}$ - sazonalidade

DMR - desvio médio real

- A terceira etapa é o cálculo do fator de crescimento ou diminuição mensal do mercado (F). No início da simulação o operador do simulador determina o valor de previsão de crescimento ou diminuição do mercado (VPM) e também determina em quais meses vai haver o crescimento ou diminuição do mercado. Se o mês previsto para a diminuição ou crescimento do mercado for maior que o mês simulado, então o fator $F$ fica igual a um. A equação 3.8 mostra o cálculo do fator F;

$$
F_{k}=\left(\frac{V P M}{100}\right)+1
$$

Onde:

$\mathrm{F}$ - fator de crescimento ou diminuição mensal do mercado

VPM - valor de previsão de crescimento ou diminuição do mercado

- A quarta etapa é cálculo da promoção de venda (PV). No início da simulação o operador do simulador determina o valor da variação percentual das vendas sobre a demanda de cada mês do ano, ou seja, determina o percentual de previsão de promoção de vendas (PPV) de cada mês do ano. A equação 3.9 mostra detalhadamente o cálculo de PV;

$$
P V_{k}=\left(\frac{P P V}{100}\right)+1
$$

Onde:

PV - promoção de venda

PPV - percentual de previsão de promoção de vendas

- A projeção da demanda real (PDR) para cada mês do horizonte de simulação é obtida a partir de todas as etapas anteriores. Após o cálculo de PDR, realiza-se o cálculo da quantidade média de peças por Calls (QPC) para cada mês do horizonte de simulação. O QPC é obtido pela média dos QPC do mesmo período da demanda histórica de 


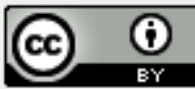

trinta e seis meses. A partir do cálculo de PDR e QPC obtém-se o número de Calls para cada mês do horizonte de simulação. As equações 3.10, 3.11 e 3.12 descrevem matematicamente o processo descrito acima;

$$
\begin{aligned}
& P D R_{k}=\left[M M_{24}+(k \times A D M)\right] \times D M R_{k} \times F_{k} \times P V_{k}, k=1,12 \\
& Q P C[36+k]=\frac{Q P C[0+k]+Q P C[12+k]+Q P C[24+k]}{3}, k=1,12
\end{aligned}
$$

Dividindo a Eq.(3.10) pela Eq. (3.11), tem-se:

$$
C[36+k]=\frac{P D R_{k}}{Q P C[36+k]}, k=1,12
$$

\subsubsection{Reposição das Peças}

A reposição da peça do CDP tem como objetivo determinar quanto e quando repor a peça. A política de reposição de peça adotada para as peças impactadas utiliza recursos dos dois principais modelos para a demanda independente que são: o modelo de revisão periódica e o modelo de revisão contínua

As peças desimpactadas não têm uma política de reposição de peças devido ao fato de não ter uma demanda significativa. Em alguns casos pode haver a necessidade de reposição de peça somente para atender as ordens em atraso (backorders).

A política de reposição de cada peça impactada sofre variações de acordo com a classificação da peça, mas basicamente o funcionamento do sistema é o seguinte:

- Quando a posição de estoque de cada peça atinge um nível mínimo (estoque mínimo), uma quantidade de peça é solicitada para repor o estoque para um nível máximo (estoque máximo). O cálculo do estoque mínimo, estoque máximo e da quantidade de peça varia de acordo com a classificação da peça (Normal ou Proteção e A, B ou C);

- Para não ocorrer falta de estoque de peça do momento em que se faz o pedido até o recebimento da peça utiliza-se um estoque de segurança (ES) obtido pela equação 3.13. Cada peça do inventário tem um valor de estoque de segurança diferente.

$$
E S=D \times \text { Fator } K
$$

Onde: 
D - desvio acumulado entre a demanda atual e a prevista

FatorK - fator de segurança relacionado ao nível de serviço desejado para a peça

\subsubsection{Nível de Serviço ao Cliente}

Para o gerenciamento do nível de serviço ao cliente utiliza-se o indicador percentual de atendimento de pedidos (PAP) que é o percentual de pedidos atendidos em sua íntegra para cada mês do horizonte de simulação. O PAP de cada peça é obtido da seguinte forma:

- Inicialmente calcula-se o número de Calls (C) no mês em que está ocorrendo a simulação, ou seja, somam-se todos os Calls do mês de simulação. Se C for igual a zero, significa dizer que não há demanda e o percentual de atendimento de pedidos (PAP) também será igual a zero. $\mathrm{Se} \mathrm{C}$ for maior que zero, calcula-se a possibilidade de atendimento de pedido (POP);

- O valor de POP é o inteiro resultante da divisão do estoque disponível total (EDT) pelo número de peças por Call (QPC) do mês em que está ocorrendo à simulação. Se o valor de POP for igual a zero, significa dizer que não há estoque disponível da peça para atender os Calls e o PAP também será igual a zero. Se o valor de POP for maior que zero, calcula-se o PAP;

- O cálculo de PAP é o resultado da divisão da POP por C. Multiplica-se o resultado por 100 para transformar o número em percentual. Se o valor de PAP for maior que 100, então, limita-se PAP a 100, ou seja, PAP $=100 \%$ (maior nível de serviço possível). O cálculo de PAP é realizado para todas as peças. As equações 3.14 e 3.15 descrevem matematicamente o cálculo de POP e PAP:

$$
\begin{aligned}
& P O P=\left[\left(\frac{E D T}{Q P C}\right)\right] \\
& P A P=\left(\frac{P O P}{C}\right) \times 100
\end{aligned}
$$

Onde:

PAP - percentual de atendimento de pedidos

POP - possibilidade de atendimento de pedido

EDT - estoque disponível total 
QPC - número de peças por Call

C - número de Calls

\subsubsection{Meses de Inventário}

Para o gerenciamento do valor investido no inventário utiliza-se o indicador meses de inventário (MI) que é a quantidade de meses que o estoque de cada peça duraria, sujeito às vendas futuras, sem que haja reposição do estoque da peça.

Quando o valor de MI é pequeno, corre-se o risco de faltar peça para o atendimento ao cliente e conseqüentemente reduzir o nível de satisfação do cliente. Quando o valor de MI é grande, corre-se o risco de ter estoques obsoletos e aumento dos custos de manutenção de estoque. A determinação do valor de MI varia de peça para peça dependendo da sua importância para o CDP.

As etapas para obtenção do MI de cada peça são as seguintes:

- Inicialmente calcula-se o estoque disponível total (EDT). Se EDT for menor que zero, atribui-se zero a EDT. Se EDT for maior que zero, calcula-se o estoque disponível total em reais (EDTR). Matematicamente tem-se:

$E D T R=E D T \times U C$

Onde:

EDT - estoque disponível total

EDTR - estoque disponível total em reais

UC - custo unitário da peça

- Após calcular o EDTR, calcula-se a média normalizada quadrimestral de vendas (MNV) de cada peça. Matematicamente tem-se:

$M V_{i}=\frac{\sum_{j=i}^{i+3} D H_{j} \times U C}{4}, i=1,12$

$N=\frac{M Q}{M M Q}$

Multiplicando a Eq. (3.17) pela Eq. (3.18), tem-se: 


$$
M N V=M V \times N
$$

Onde:

MNV - média normalizada quadrimestral de vendas

MV - média de vendas mensal

$\mathrm{N}$ - normalização

DH - demanda histórica mensal

MQ - média quadrimestral dos dias úteis

MMQ - média das médias quadrimestrais dos dias úteis

MI - meses de inventário

- A ultima etapa é a realização do cálculo dos meses de inventário (MI). Se EDTR ou MNV for igual a zero, MI também será igual a zero. Se EDTR e MNV forem maiores que zero, o MI é obtido da seguinte forma:

$M I=\frac{E D T R}{M N V}$

\subsubsection{Scrap}

As peças são consideradas Scrap quando não podem ser usadas para o seu propósito original, mas têm certo valor para o inventário. As etapas para o cálculo das peças Scrap são:

- Inicialmente verifica-se se a peça é Scrap e adiciona a mesma na lista de peças Scrap. Se a lista de peças Scrap já tiver alguma peça, então as peças são ordenadas segundo uma regra de prioridade que pode ser definida: por maior peso da peça, por menor peso da peça, por maior custo unitário da peça, por menor custo unitário da peça;

- Na próxima etapa, calcula-se o valor monetário do estoque de cada peça (VEP) candidata a Scrap. Após calcula-se a cota mensal de Scrap (CMS). A cota anual de Scrap (CAS) é determinada pelo operador do simulador no início da simulação na entrada de dados geral. A equações 3.21 e 3.22 mostram o cálculo do VEP e do CMS:

$V E P=P F E \times U C$

$C M S=\frac{C A S}{12}$ 
Onde:

VEP - valor do estoque de cada peça

PFE - posição final do estoque

UC - custo unitário da peça

CMS - cota mensal de Scrap

CAS - cota anual de Scrap

- Se o valor do estoque de cada peça (VEP) for menor ou igual a cota mensal de Scrap (CMS), desconta-se o valor do VEP da CMS. No indicador de quantidade de Scrap da peça (QSP) no mês atual de simulação é colocado o valor do VEP e no estoque físico da peça novo $\left(\mathrm{DPF}_{\text {novo }}\right)$ coloca-se zero. Se após esse procedimento ainda houver saldo na $\mathrm{CMS}_{\text {novo, }}$, aplica-se o mesmo procedimento na próxima peça. Se não houver mais saldo na $\mathrm{CMS}_{\text {novo, }}$ zeram-se os marcadores de candidato a Scrap. Matematicamente tem-se:

Se $\mathrm{VEP} \leq \mathrm{CMS}:$

$C M S_{\text {novo }}=C M S-V E P$

$Q S P=V E P$

$D P F_{\text {novo }}=0$

- Se o valor do VEP for maior que a CMS, calcula-se o QSP, CMS e o DPF. Matematicamente tem-se:

Se VEP > CMS

$$
\begin{aligned}
& Q S P=\frac{C M S}{U C} \\
& C M S_{\text {novo }}=0 \\
& D P F_{\text {novo }}=D P F-Q S P
\end{aligned}
$$

\subsubsection{Devolução de Peças}

A devolução da peça é realizada quando a mesma não apresenta uma demanda histórica condizente com seu estoque. A devolução ocorre quando o estoque da peça é maior 
do que a demanda histórica dos últimos trinta e seis meses. No início da simulação o operador do simulador determina a freqüência para devolução das peças. A freqüência pode ser mensal, bimestral, trimestral, quadrimestral, semestral ou anual.

$\mathrm{Na}$ operação de devolução de peças, o CDP já desembolsou uma quantia monetária (valor da peça e impostos) para ter aquela peça em seu estoque, com isso é necessário calcular a quantidade de devolução e o valor em reais dessa quantidade de devolução. O cálculo da quantidade para devolução e do valor de devolução é realizado da seguinte forma:

- Nos meses determinados como de devolução, verifica-se o valor total disponível para devolução em reais (VTD). Seleciona-se a peça caso sua quantidade no estoque físico da peça (DPF) seja maior que zero. Calcula-se a demanda histórica (DH) dos últimos trinta e seis meses da peça e compara-se com a quantidade do DPF da peça. Se a quantidade do DPF da peça for maior que a DH dos últimos trinta e seis meses da mesma, calcula-se a quantidade de peça para devolução (QPD). Após calcular QPD, calcula-se o valor de devolução da peça (VDP) em reais. Matematicamente tem-se:

Se $D P F>0$ e $D P F>\sum_{1}^{36} D H_{i}$ :

$Q P D=D P F-\sum_{1}^{36} D H_{i}$

$V D P=U C \times Q P D$

Onde:

$\mathrm{DPF}$ - estoque físico da peça

DH - demanda histórica

QPD - quantidade de peça para devolução

VDP - valor de devolução da peça

UC - custo unitário da peça

- Se o valor de devolução da peça (VDP) for menor que o valor total disponível para devolução (VTD) em reais, então o novo VTD será igual à diferença entre o VTD e o VDP e o novo estoque físico da peça (DPF) será igual à DH dos últimos 36 meses. O que restou de VTD é utilizado na próxima peça. Matematicamente tem-se: 
$V T D_{\text {novo }}=V T D-V D P$

$D P F_{\text {novo }}=\sum_{1}^{36} D H_{i}$

- Se o valor de devolução da peça (VDP) for maior ou igual ao valor total disponível para devolução (VTD), então a quantidade de peça para devolução (QPD) será igual à divisão de VTD pelo custo unitário (UC), pois só pode ser realizada a devolução da quantidade de peça referente ao VTD. O novo VTD será igual a zero e o novo DPF será igual ao DPF menos a QPD. Matematicamente tem-se:

$$
\begin{aligned}
& \text { Se VDP } \geq \text { VTD: } \\
& Q P D_{\text {novo }}=\frac{V T D}{U C} \\
& V T D_{\text {novo }}=O \\
& D P F_{\text {novo }}=D P F-Q P D
\end{aligned}
$$

\subsubsection{Previsão de Demanda}

A previsão de demanda é realizada em todo final do mês de simulação com o objetivo de prever o mês seguinte. A previsão de demanda é realizada baseada no método de suavização exponencial simples. Para realizar cálculo da demanda mensal estimada (DME) do próximo mês de simulação utiliza-se a demanda real atual, a previsão de demanda atual e o coeficiente de suavização $(\alpha)$.

A demanda real e a previsão de demanda no primeiro mês de simulação são fornecidas pelo arquivo peça. Após o primeiro ciclo de simulação utiliza-se a demanda real projetada e utiliza-se a DME calculada no primeiro ciclo de simulação e assim por diante em todos os meses de simulação até completar o horizonte de simulação. O coeficiente de suavização é determinado pelo operador do simulador para cada fonte de peça (nacional ou importada) no início da simulação e seu valor deve estar entre zero e um. Na equação 3.36 tem-se matematicamente o cálculo da DME:

$$
D M E=\left(\left(\alpha \times D_{\text {real }}\right)+(1-\alpha) \times D_{\text {prev }}\right)
$$


Onde:

DME - previsão de demanda para o próximo mês ou demanda mensal estimada

$\mathrm{D}_{\text {real }}$ - demanda real do mês atual

$\mathrm{D}_{\text {prev }}$ - previsão de demanda do mês atual

$\alpha$ - coeficiente de suavização

Além da previsão de demanda, realiza-se o monitoramento da previsão de demanda e reclassifica-se a peça quando necessário. O monitoramento é realizado com o cálculo do desvio acumulado (D). O D é também calculado no final de cada mês. Para o calculo do desvio acumulado do primeiro mês de simulação utiliza-se o desvio fornecido pelo arquivo peça. Após o primeiro ciclo de simulação utiliza-se sempre o desvio anterior (DA), e assim por diante em todos os meses de simulação até completar o horizonte de simulação. Além do desvio anterior, o desvio acumulado (D) é composto pelo erro ponderado (EP). O EP e o D são obtidos pelas equações 3.37 e 3.38 .

$$
\begin{aligned}
& E P=\left(\left(D_{\text {real }}-D_{\text {prev }}-D A\right) \times \alpha\right) \\
& D=E P+D A
\end{aligned}
$$

Onde:

D - desvio acumulado

$\mathrm{EP}$ - erro ponderado

DA - desvio anterior

A última etapa é verificar a necessidade de reclassificar a peça e definir o momento necessário para realizar a reclassificação. No simulador há três tipos de reclassificação:

- Com relação à análise: a peça pode ser impactada ou desimpactada.

- Com relação à classe: a peça pode ser classificada como A, B ou C;

- Com relação à classificação proteção e à classificação normal.

\subsection{Dados de Saída do Simulinve}

O simulador oferece a possibilidade da saída de dados em planilha do Microsoft Excel® ou via interface do próprio simulador. Os dados de saída do simulador são:

- Código do Inventário: é o código utilizado para o gerenciamento do inventário; 
- Código da Peça: é o código da peça utilizado para localização da peça no inventário;

- Família: é a família à qual a peça pertence;

- Número de Calls: é a quantidade de Calls de cada mês do horizonte de simulação;

- Quantidade de Peças por Calls: é a quantidade de peças por Calls de cada mês do horizonte de simulação;

- Backorders: são os Calls de cada peça que não foram atendidos durante cada mês do horizonte de simulação;

- Reposição e Recebimento da Peça: é quantidade a ser reposta e recebida de cada peça durante o horizonte de simulação;

- Meses de Inventário: é a quantidade de meses de inventário de cada peça durante o horizonte de simulação;

- Percentual de Atendimento de Pedidos: é o percentual de atendimentos de pedidos de cada peça durante o horizonte de simulação;

- Estoque das Peças: é o estoque das peças durante o horizonte de simulação;

- Devolução das Peças: é a quantidade devolvida aos fornecedores das peças pertencentes durante o horizonte de simulação;

- Scrap: é a quantidade de peças Scrap em cada mês do horizonte de simulação;

- Previsão de Demanda: é a previsão de demanda estimada da peça em cada mês do horizonte de simulação;

- Desvio: é o desvio acumulado entre a demanda real e a demanda prevista da peça em cada mês do horizonte de simulação.

\section{Considerações Finais}

Este trabalho apresentou um simulador de inventário para um CDP que permite fazer previsões de demanda, da política de reposição das peças, do percentual de atendimento dos pedidos, do valor do inventário, da quantidade de Scrap, da quantidade de peças para devolução, sempre com a preocupação de interagir de forma flexível e mantendo a integridade dos dados e variáveis originais.

A realização deste trabalho evidenciou algumas vantagens do uso do simulador de inventário para o CDP em estudo, que foram: 
- A possibilidade de avaliar diferentes políticas gerenciais do inventário do CDP sem impactar ou interromper os processos em andamento no mundo real, o que poderia produzir custos adicionais significativos;

- A possibilidade de comprimir o tempo, permitindo aos responsáveis pelo inventário conhecer em pouco tempo, as conseqüências de longo prazo de suas ações;

- A possibilidade de melhor entender o funcionamento dos processos gerenciais do inventário do CDP, assim como suas influências sobre o sistema como um todo;

- A observação da importância de cada peça do inventário, assim como a sinalização de quando a peça deve ser descontinuada ou devolvida ao fornecedor;

- A possibilidade de melhor avaliar o desempenho do gerenciamento do inventário;

As principais dificuldades encontradas durante a realização do trabalho foram:

- A coleta de dados ocorreu com dificuldade devido o CDP em estudo ter um política de privacidade de dados;

- Realização da projeção da demanda futura das peças.

Com a utilização do simulador, os gestores podem alcançar um melhor desempenho no gerenciamento do inventário. A partir de simulações, os responsáveis pelo inventário têm condições de tomar decisões com maior segurança e mais rapidamente.

\section{REFERÊNCIAS}

BANKS, J. Handbook of simulation: principles, methodology, advances, applications, and practice. New York: John Wiley \& Sons, 1998.

BANKS, J.; CARLSON, J. S.; NELSON, B. L. Discrete-event system simulation. 2.ed. New Jersey: Prentice-Hall, 1996.

BERTRAND, J. W. M; FRANSOO, J. C. Modeling and simulation: operations management research methodologies using quantitative modeling. International Journal of Operations \& Production Management, v.22, n.2, p.241-264, 2002.

BOWERSOX, D. J.; CLOSS, D. J. Logística empresarial: o processo de integração da cadeia de suprimento. São Paulo: Atlas, 2001.

BREWER, A.M.; BUTTON, K. J.; HENSHER, D. A. Handbook of logistics and supplychain management. Kidlington: Elsevier Science Ltd, 2001. 


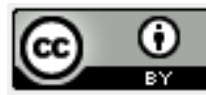

CARSON II, J, S. Introduction to modeling and simulation. Proceedings of the 2004 Winter Simulation Conference, p.01-08, 2004.

CENTENO, M. A.; CARRILLO, M. Challenges of introducing simulation as a decision making tool. Proceedings of the 2001 Winter Simulation Conference, p.17-21, 2001.

COX III, J. F.; BLACKSTONE JUNIOR, J. H. APICS Dictionary. 9.ed. Falls Church: American Production and Inventory Control Society, 2002.

DEAR, A. Inventory management demystified. London: Chapman and Hall, 1990.

FARAH JUNIOR, M. Os desafios da logística e os centros de distribuição física. Revista FAE BUSINESS, n.2, p.44-46, 2002.

FRAZELLE, E. World-class warehousing and material handling. New York: McGrawHill, 2002.

FORGATY, D. W.; BLACSTONE, J.H.; HOFFMANN, T. R. Production \& inventory management. Cincinnati: South Western Publishing Co, 1991.

FREITAS FILHO, P. J. Introdução à modelagem e simulação de sistemas: com aplicação em Arena. Florianópolis: Visual Books, 2001.

GAITHER, N.; FRAIZER, G. Administração da produção e operações. 8.ed. São Paulo: Pioneira, 2002.

HARREL, C.; TUMAY, K. Simulation made easy. IIE Solutions, p.39-41, July, 1997.

KELTON, W. D.; SADOWSKI, STURROCK, D. T. Simulation with arena. New York: WCB/McGraw Hill, 1998.

KRAJEWSKI, L. J; RITZMAN, L. P. Administração da produção e operações. São Paulo: Prentice Hall, 2004.

MULCAHY, D. E. Warehouse distribution \& operations handbook. New York: McGrawHill, 1994.

PRITSKER, A. A. B. Introduction to simulation and SLAM II. New York : John Wiley \& Sons, 1986.

RODRIGUES, G. G; PIZZOLATO, N. D. Centros de Distribuição: armazenagem estratégica. XXIII Encontro Nacional de Engenharia de Produção, Ouro Preto-Minas Gerais, p.01-08, 2003.

SLACK, N.; CHAMBERS, S.; HARLAND, C.; HARRISON, A.; JOHNSTON, R. Administração da produção. São Paulo: Atlas, 1997. 
SHANNON, R. E. Introduction to the art and science of simulation. In: Proceedings of the 1998 Winter Simulation Conference. p. 07-14, 1998.

TOMPKINS, J. A.; WHITE, J. A.; BOZER, Y. A.; TANCHOCO, J. M. A. Facilities planning. 2. ed. New York: John Wiley \& Sons, 1996.

TUBINO, D. F. Manual de Planejamento e controle da produção. São Paulo: Atlas, 2000.

VAN DEN BERG, J. P.; ZIJM, W. H.M. Models for warehouse management: classification and examples. International Journal of Production Economics. n.59, p.519$528,1999$.

O trabalho relatado neste artigo teve apoio do CNPq e da CAPES. 